\title{
Visualizing computation in large-scale cellular automata
}

\author{
Hugo Cisneros ${ }^{1}$, Josef Sivic ${ }^{1,2}$, Tomas Mikolov ${ }^{1}$ \\ ${ }^{1}$ CIIRC - Czech Institute of Informatics, Robotics and Cybernetics, Czech Technical University in Prague. \\ ${ }^{2}$ WILLOW project, Département d'Informatique de l'École Normale Supérieure \\ ENS/INRIA/CNRS UMR 8548, PSL Research University.
}

\begin{abstract}
Emergent processes in complex systems such as cellular automata can perform computations of increasing complexity, and could possibly lead to artificial evolution. Such a feat would require scaling up current simulation sizes to allow for enough computational capacity. Understanding complex computations happening in cellular automata and other systems capable of emergence poses many challenges, especially in large-scale systems. We propose methods for coarse-graining cellular automata based on frequency analysis of cell states, clustering and autoencoders. These innovative techniques facilitate the discovery of large-scale structure formation and complexity analysis in those systems. They emphasize interesting behaviors in elementary cellular automata while filtering out background patterns. Moreover, our methods reduce large 2D automata to smaller sizes and enable identifying systems that behave interestingly at multiple scales.
\end{abstract}

\section{Introduction}

Cellular automata (CA) have been extensively studied since the 1960s. Originally designed and studied to create artificial evolution from self-replication (Von Neumann and Burks, 1966, Langton, 1984), previously studied cellular automata simulations were often of relatively modest sizes. Only specific rules with repetitive or predictable dynamics such as John Conway's Game of Life (Gardner, 1970) have been scaled up to larger grid sizes $\left(10^{4} \times 10^{4}\right.$ or more cells).

For complex phenomena such as artificial evolution to exist and be open-ended within those simulated worlds, there needs to be sufficient "capacity" - a large enough statespace. In nature, complex and significantly different dynamics often arise from uniform laws at a smaller scale (Anderson 1972). It seems unlikely that such complex processes, like artificial evolution, could happen in too small CAs because higher order dynamics do not have enough capacity to emerge. However, several issues arise when scaling CAs to large sizes:

- Time complexity rapidly becomes a bottleneck. Updating a large number of cells is costly. Tricks such as caching of some of the computations can help, but do not always improve performance significantly (Gosper, 1984).
- Memory complexity can also become an issue when dealing with numerous states, and especially grids in 3 dimensions and more. In that case, even the underlying rule of the system cannot be stored within reasonable memory capacity.

- Visual inspection of these large grids is infeasible. Studying CA complexity is rendered difficult by the highly variable nature of emergent processes. It is especially the case for large-scale systems.

When working with such large systems, it is less relevant to focus on the local behaviors at the single cell level. This is similar to other complex systems like the weather, in which behaviors of individual atoms in a cloud are irrelevant to large-scale air mass movements. Much richer behaviors can be observed from studying large patterns' formation and their evolution. This should also hold true for CAs; we further discuss this question in Conclusion In this paper,
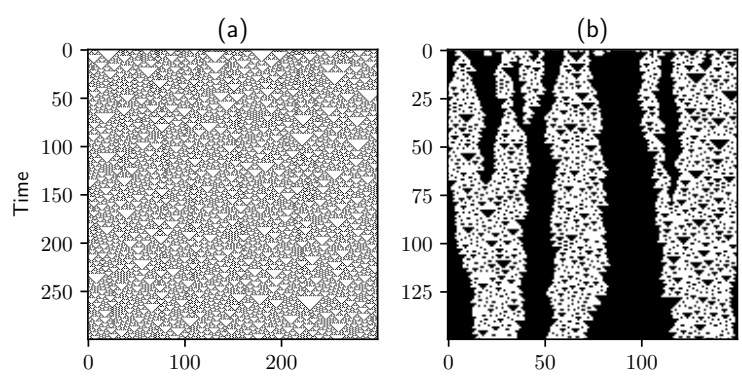

Figure 1: Hidden structures in rule 18 are uncovered by filtering the space-time diagram with our frequency histogram-based method. (a) shows 300 timesteps of a randomly initialized rule 18 simulation. Notice the complex structures made visible in (b) with our method.

we investigate techniques which can help us visualize large space-time diagrams of CAs. We demonstrate that simple clustering and coarse-graining techniques can be used in order to perceive structures which cannot emerge on smaller grids. This is also useful for disordered cellular automata with hidden structures as it is the case for the elementary cellular automaton rule 18, illustrated in Figure 1 - more details in Results 
Reducing large grids to smaller sizes while preserving interesting behaviors such as pattern formation is essential to apply to these CAs complexity metrics designed to work on modestly sized grids (Grassberger, 1986, Zenil, 2010, SolerToscano et al., 2014; Zenil et al., 2015). Common metrics of complexity are often limited by the number of components in the systems (number of cells in a CA grid, timesteps, etc.) or may not be effective when small scale patterns are less relevant than large-scale ones.

\section{Related work}

Previous work on coarse-graining cellular automata focused either on conserving the main computational properties of $\mathrm{CA}$ rules through exact coarse-graining or on filtering interesting behaviors without reducing the amount of computations. Our work both highlights interesting behaviors and compresses the representation, which we argue are necessary to study complexity in large cellular automata.

\section{Coarse-graining in cellular automata}

Coarse-graining is an approximation procedure used to speed up computations in systems made of many components. It originated in Levitt and Warshel (1975) and is now widely used in physics to model complex systems at various granularity levels, and is successful at modeling biomolecules (Potoyan et al., 2013, Ingólfsson et al., 2014, Kmiecik et al. 2016).

Exact coarse-graining of elementary cellular automata (ECA) has been investigated extensively in Israeli and Goldenfeld (2004, 2006). Authors found ways of rewriting one-dimensional $\mathrm{CA}$ rules into each other through coarsegraining of the transition rule. They built a graph of equivalence of all 256 ECA and identified some rules that do not admit any computational reduction. This indicates that some cellular automata are accomplishing fundamentally more computations than others.

\section{Filtering}

Filtering cellular automata (CA) was introduced to reduce a CA's behavior to its most relevant parts. The goal is to extract relevant irregularities from a CA's space-time diagram. Seminal work by Hanson and Crutchfield (1992, 1997) formalized the notion of domains and coherent structures in cellular automata. They used a set of regular languages to represent cellular automata dynamics and extract relevant behaviors such as discontinuities between regular domains or "particles". Figure 7 shows a filtering example for cellular automaton rule 110 - in Wolfram's numbering.

A filtering method similar to our proposed frequencybased coarse-graining - originally presented as a complexity metric for cellular automata — is introduced in Wuensche (1999). The author proposes to progressively filter out cells in cellular automata's space-time diagrams according to read frequency of the rule table. Cells that originated from frequent rule table lookups are set to a quiescent or null state. The choice of threshold has to be decided by a user for each rule. Another notable difference is the method aims at making visualization of gliders easier without reducing the size of the grid or making more compact representations.

More recent work by Shalizi et al. (2006) uses the combination of a modified Lyapunov exponent approach with statistical complexity (Shalizi et al. 2004) to underline complex behaviors. However, the first method requires repeated perturbations and simulations of the system to study its sensitivity.

\section{Scaling-up cellular automata}

Hashlife (Gosper, 1984) and other Game of Life-specific optimizations enable simulating a large number of cells for numerous timesteps. Nonetheless, these algorithms essentially exploit input redundancy. The regularity in patterns allowing such optimizations might indicate a lack of novel patterns being generated by the system.

This also means that Game of Life-based simulations are computationally reducible to a much simpler system, indicating that its computations are inefficient (Wolfram, 2002). An optimally complex-behaving computational model should be impossible to predict except when computing its actual evolution step by step.

In the following, we used coarse-graining as a method for scaling down CAs in both time and space in order to make visualization of larger patterns and complex behaviors easier. The underlying fine-scale computations may be essential for these larger patterns to appear, hence the necessity to keep them. However, analogous to many natural processes (swarms, chemistry, cells in an organism, DNA), interesting behaviors might not be observable at the level of individual components - or small groups of components (individuals, single cells or molecules in the examples above). We view coarse-graining as a way to reduce a cellular automaton's space-time diagram to its most relevant parts while keeping primary dynamics in the background. The resulting diagram would ideally be an irreducible system.

\section{Proposed coarse-graining of cellular automata}

For reasons stated above, we introduce coarse-graining methods for cellular automata that are not reversible - information is discarded in the process. This process does not attempt to find shortcuts for the computations of a cellular automaton, but rather to selects relevant parts of the spacetime diagram and discards information irrelevant to the core behavior. For example, a standard glider in Game of Life spanning $3 \times 3$ cells could be replaced with a single cell moving diagonally when coarse-graining by a factor 3 . This is because the actual oscillator's dynamics might not be relevant at this coarser scale.

Coarse-graining is akin to constructing supercells from blocks of individual cells. These supercells are assigned a 
new state and form a coarser partitioning of the initial grid which can be studied as its own system. In particular, complexity metrics or further coarse-graining can be applied to this new grid.

\section{Frequency histogram coarse-graining}

A simple coarse-graining is achieved by mapping blocks to a single supercell state according to the probability of this configuration appearing, given a previously constructed model. The easiest way to think of it is with a simple frequency counting model of the distribution of $2 \times 2$ blocks in a $2 \mathrm{D}$ CA. For a 2 -state automaton, there are 16 possible supercell configurations. The simplest model for the occurrence of these blocks is their empirical frequency. Let us consider a CA with $N$ blocks of $2 \times 2$ cells, let $S^{(i n)}=$ $\{0000,0001,0010, \ldots, 1111\}$ be the set of $2 \times 2$ blocks and $s_{i} \in S^{(i n)}$ be a given supercell. The probability $p_{i}$ of observing supercell $i$ on a grid $G$ is estimated with

$$
p_{i}=\frac{\operatorname{count}_{G}\left(s_{i}\right)}{\sum_{j \in S^{(i n)}} \operatorname{count}_{G}\left(s_{j}\right)}
$$

where count ${ }_{G}\left(s_{i}\right)$ is the number of blocks matching $\left(s_{i}\right)$ in $G$.

Supercells can then be assigned a particular state. We call the corresponding mapping $f: S^{(i n)} \mapsto S^{(o u t)} . S^{(o u t)}$ can be chosen depending on the desired output or use. For instance, with $S^{(\text {out })}=\{0,1\}$ we can define $f$ to map each supercell $s_{i}$ as follows:

$$
f(i)= \begin{cases}0 & \text { if } p_{i} \geq \alpha \\ 1 & \text { if } p_{i}<\alpha\end{cases}
$$

where $\alpha$ is a chosen threshold.

Partitioning the histogram. This method can be understood as partitioning the histogram of supercell frequency. In equation (2), supercells with low probability - with higher self-information - are mapped to state 1 whereas commonly occurring states are mapped to 0 .

Choosing a partition of the histogram is equivalent to selecting a suitable $\alpha$ - scalar for two output states, or vector $\alpha=\left(\alpha_{1}, \ldots, \alpha_{n}\right)$ for $n$ output states. Therefore, one can map supercells to any number of target states (three or more) by partitioning the frequency histogram into any number of bins. Supercell distribution can be anything between uniform and very unbalanced, with a few supercells being overwhelmingly represented (background) and only a few occurrences of other configurations. The chosen partitioning has to deal with both situations equally well. In the following, we use a uniform partitioning of the area under the negative log-histogram for elementary cellular automata supercells are divided into two bins of equal summed negative logarithmic probability. For 2D CAs, we use the same method but with quadratic partition of the histogram $\left(1 / k^{2}\right.$ instead of $1 / k$, with $k$ the number of output states, chosen because of better visual results).

Dithering. Histogram partitioning introduces another set of parameters to be manually tuned, adding complexity to the procedure. An alternative way to produce an output image from the histogram is to use dithering. Dithering is an image processing technique commonly used to reduce large visual artifacts induced by quantization errors. Noise is added to the image during the quantization process to make the average local value of a set of pixels as close to their target continuous value as possible. The resulting image is created so as to match target continuous values with discrete values only - cell states in the grid. It can be seen as another way of partitioning the histogram with variable thresholds that depend on a running quantization error. Figure 2 shows a comparison of dithering and regular histogram partitioning (Floyd-Steinberg's algorithm was used (Floyd and Steinberg, 1976).

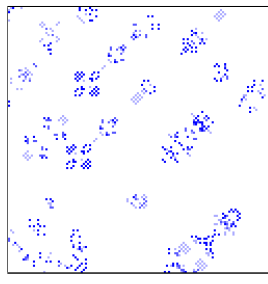

(a) Original CA

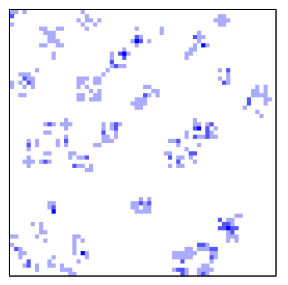

(b) w/out dithering

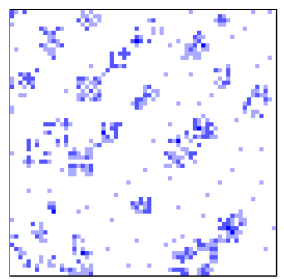

(c) with dithering
Figure 2: Close-up view of coarse-graining effects on a 4-states CA rule (1 shade of blue per state). Both coarsegraining methods conserve many of the interesting structures. Dithering introduces additional artifacts on regular backgrounds. Fig. 2 a shows actual states in the CA simulation on a $128 \times 128$ grid. Fig. $2 \mathrm{~b}$ is a coarse-grained version of $2 \mathrm{a}$ with histogram coarse-graining, the grid is $64 \times 64$ cells $2 \mathrm{c}$ is obtained with histogram coarse-graining and dithering (see Dithering).

Visualization. One advantage of this frequency histogram-based method is that it naturally highlights rarer events in the simulation grid, creating a "heatmap" of the simulation's activity. Since we sort supercells according to their observed frequency, the right choice of colors e.g. progressively darker gradient — can lead to automatic highlighting of active regions of a cellular automaton. Figure 3 shows the same simulation both unprocessed and downscaled by a factor of 4 with coarse-graining. Although much coarser, Figure $3 b$ is more readable than the base version, which is helpful when dealing with large grids 11

\footnotetext{
${ }^{1}$ Several figures in this paper have animated versions, accessible at the paper's project page https://hugocisneros.com/ ALIFE-Paper-2020/
} 


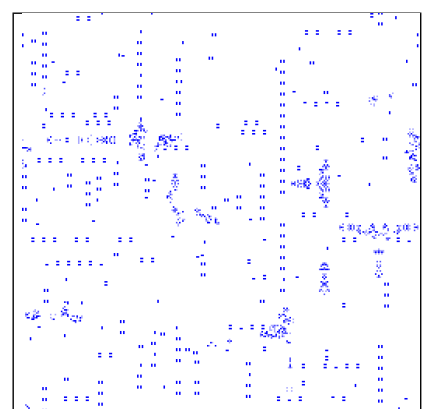

(a) Base grid

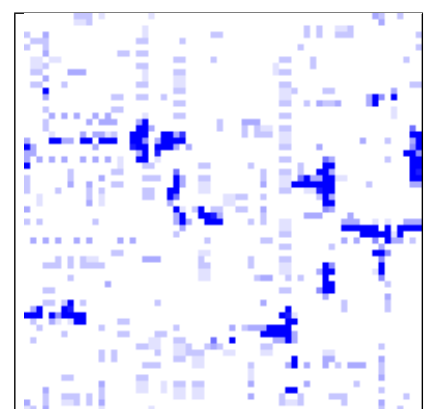

(b) Coarse-grained
Figure 3: Side-to-side comparison of a CA simulation and its coarse-grained version. The first simulation is $256 \times$ 256 cells and the second has been coarse-grained to $64 \times$ 64 . Notice the interesting patterns on Figure $3 a$ are hardly distinguishable. They are highlighted by histogram-based coarse-graining in Figure $3 b$

Hierarchical coarse-graining. The above procedure can be applied recursively to the same cellular automaton or with larger block sizes to get a progressively coarser representation. Since information is systematically discarded in the process, it cannot be applied any number of times. For this reason, many 2D CAs exhibiting interesting behaviors at the micro-level but not at the macro-level have no remaining visible structure after reducing their scale several times with this method.

Because a simple model like frequency counting can be estimated quickly, hierarchical coarse-graining is easily applied to large grids, reducing the size by a factor of $n$ (block size) every time. For instance, this property makes it suitable to search the cellular automata rule space for CAs behaving interestingly at multiple coarse-graining levels simultaneously.

\section{Clustering}

Another way to convert blocks of cells for coarse-graining is to distribute these blocks into a small number of clusters, where each group becomes the new coarse state.

Several distance functions may apply here, the most natural of which being Hamming distance, which measures how many states differ between two positions (Hamming, 1950). It is defined for two strings of equal length $n$, $s_{1}=\left[s_{(1,1)}, \ldots, s_{(1, n)}\right]$ and $s_{2}=\left[s_{(2,1)}, \ldots, s_{(2, n)}\right]$, as the number of positions where the two strings differ:

$$
\sum_{k=1}^{n} \mathbb{1}\left\{s_{1}^{(k)} \neq s_{2}^{(k)}\right\}
$$

A supercell of $N \times N$ cells of a CA can be converted into a string to be compared to other blocks with the Hamming distance. For CAs, we limit ourselves to strings of digits representing states, i.e. $s_{(i, j)} \in \mathbb{N}$. We use a vanilla implementation of the K-means algorithm where clusters' centers are computed using a continuous average of position vectors rounded to nearest integer values. Clusters are initialized with randomly selected observations.

\section{Autoencoders for coarse-graining}

Instead of just relying on the amount of information of a given supercell's configuration, one can also try to automatically find a relevant representation with dimensionality reduction methods. Autoencoders are neural networks composed of an encoder part and a decoder part, originally designed to identify principal components of a collection of data points (Baldi and Hornik, 1989, Hinton, 1989, Kramer. 1991). An encoder neural network converts data to a latent vector of smaller dimension than the original input. Then, a decoder neural network reconstructs a vector with the same dimension as the input from this encoded latent representation.These models can automatically find an optimal constrained representation through minimizing a reconstruction loss between the original input and the reconstructed output.

We denote the encoder network with $E$ and the decoder network with $D$. We frame the reconstruction problem as a $N$ class classification problem with multiple components - one class per input state, one component for each cell of the $K$ cells in a block. The reconstruction loss is the component-wise cross-entropy between the state of each input cell and the reconstructed state after $D \circ E$ is applied.

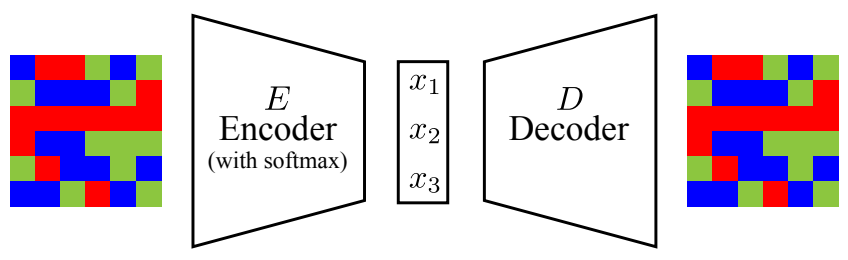

Figure 4: Diagram of the autoencoder architecture used for coarse-graining. A block of $6 \times 6$ cells is encoded in a vector of fixed dimension. There are 3 components in the example. They can either represent a RGB color or a 3 states smaller automaton.

Figure 4 illustrates the autoencoder layout for coarsegraining. By adjusting the block size and dimension of the encoded vector, one can influence the amount of information conserved during encoding. Naturally, smaller blocks will be more easily represented in lower dimension.

The encoder has a softmax layer to ensure the coded state's components sum to one. Therefore, one can view this coded supercell as a mixture of states which can either be kept as is or converted to a discrete state by keeping the maximal component only. They are trained with stochastic gradient descent until convergence. 


\section{Results}

We evaluate our proposed coarse-graining methods in the following two different ways:

- We compare our results on elementary cellular automata (ECA) to previous works on particle and domain filtering.

- We use a metric which evaluates complexity of CAs introduced in Cisneros et al. (2019) in order to compare our methods' complexity metric scores of the coarse-grained systems and contrast the scores against a standard image processing baseline that computes local average of neighbouring cells followed by downscaling the grid. Using the complexity metric we measure to what extent the interesting behavior of cellular automata is conserved after coarse-graining compared to this image processing baseline.

In the following we begin by showing that a simple histogram-based coarse-graining is effective at detecting structures (such as gliders) in ECA space-time diagrams. Our method achieves results comparable with previous work, while being simpler to apply.

\section{Domains and filtering}

In the space-time diagrams of cellular automata, moving structures such as gliders are embedded in uniform or periodic backgrounds, or "domains". This domain is different depending on the rule: some ECAs have uniform backgrounds, checkerboard backgrounds or more complicated patterns (e.g. rule 110). Crutchfield and Hanson (1993) also identified chaotic domains, which cannot support regular gliders but have "walls" and "particles". Those correspond, respectively, to boundaries between two chaotic domains and propagating defects (localized structures with a pattern different from the domain) within a domain.

Our proposed coarse-graining methods offer interesting perspectives to filter cellular automata's space-time diagrams, which enables identifying gliders and studying the formation of large-scale patterns. We find that a simple histogram coarse-graining achieves results comparable to those reported in Hanson and Crutchfield (1992); Eloranta and Nummelin (1992); Hanson and Crutchfield (1997); Wuensche (2011) for ECA rules 18 and 54. A similar approach was undertaken in (Wuensche, 1999) in which the authors used the entropy of rule table lookup frequencies to filter out regular domains in the space-time diagrams of cellular automata and to identify gliders and domain boundaries. However, Wuensche's approach described in Wuensche (1999) does not attempt to downscale space-time diagrams.

\section{Results on elementary cellular automata}

We apply frequency histogram-based coarse-graining on elementary cellular automata (ECA) and obtain space-time diagrams with suppressed background domains. Resulting (a)

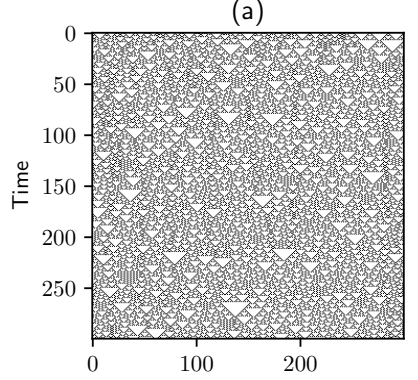

(c)
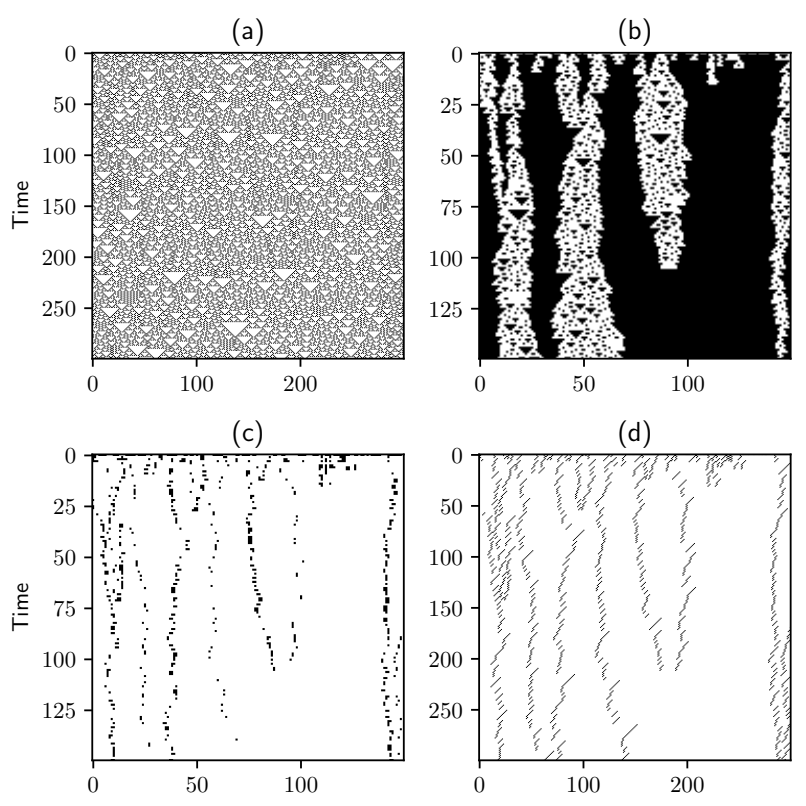

(d)

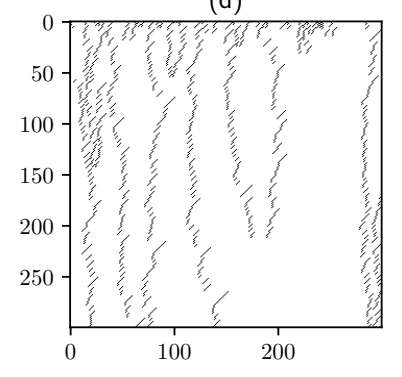

Figure 5: Space-time diagrams for rule 18 in elementary cellular automata. (a) Standard rule 18 space-time diagram, starting from a random position. (b) Filtered domain with our frequency coarse-graining (even). (c) Our domain boundaries extracted from the filtered domains in (b). (d) Domain boundaries computed according to (Hanson and Crutchfield, 1992). Note that (a) shows semi-chaotic behaviour, which is hard to interpret, whereas our method (b) highlights distinct domains within the disordered spacediagram in (a). The detected domains and domain boundaries from previous work (d) and ours (c) are very similar.

partitions of ECAs' space-time diagram are similar to results reported by Hanson and Crutchfield (1992, 1997). Figure 5 (a) and 6(a) show the space-time diagrams of rules 18 and 54 with random initialization. Boundaries between different background patterns in both Figures were obtained with coarse-graining; they are similar to boundaries obtained by Hanson and Crutchfield. We also observe the propagation of many of the same particles and defects without any prior information about the cellular automaton rule.

Particles in Rule 54 have been used to implement computations (Boccara et al., 1991; Pivato, 2007; Martinez et al. 2014). Because the presented reduction reduces the size of the grid, it can merge some of those particles, sometimes resulting in ambiguities and gaps. However, our goal here is not to precisely describe particle interactions in order to manipulate or construct complex computations manually. Underlying computations described in the works above are still happening within our reduced CA simulation. We consider the apparent destruction of some of these fine-scale details acceptable in order to discover larger-scale complex behav- 
ior.

Our method is also arguably much simpler than computational mechanics (used by Hanson and Crutchfield) which requires some reverse-engineering of the rule and the construction of a finite-state transducer to generate output symbols. Although full automation has been demonstrated, this method introduces significant overhead (Rupe and Crutchfield, 2018). On the other hand, our method is sensitive to the quality of statistical estimation of the frequency histogram (see equation (1) ) and needs enough input examples to achieve a reasonable result - examples in Figure 5 and 6 used simulations with the width of 3000 cells, ran for 6000 timesteps to obtain reliable pattern frequency estimates.

In the Figures, we used the coarse-graining method introduced in Frequency histogram coarse-graining Space-time diagrams are coarse-grained by a factor 2 to a binary automaton - each cell corresponds to a 2-cell block. These binary coarse-graining results in the Figures are labeled (c). Because of the statistical nature of the domains of these 1D ECA's and the use of blocks of size 2, filtered domains differ depending on the starting position of coarse-graining. We distinguish an odd and even filtered domain.

Figure 5(c) is obtained by applying the element-wise $O R$ operator to both the even and odd domain diagrams to merge them into a single space-time diagram. Figure 6(c) is obtained by computing differences between neighboring cells after the filtering process to highlight lines. Figure 7 is another example showing filtering of particles in rule 110 .

\section{Complexity metrics and coarse-graining}

Coarse-graining is not only useful for detecting gliders and domains in space-time diagrams, but also as a tool to visualize large CAs. To evaluate the quality of our proposed coarse-graining methods, we compare complexity scores computed according to Cisneros et al. (2019) for different coarse-graining methods. This metric was shown to correlate well with a user study for interesting automata. It uses neural networks to estimate how easy it is to learn a compressed representation of a CA. We also compute the scores on downscaled CAs as a baseline. Local averaging is used for downscaling, with each block of $N$ cells being replaced by their average value rounded to the nearest integer state.

Experiments begin by sampling 3600 cellular automata rules with 3 or 4 states. We apply the complexity metric on a randomly initialized simulation on a $512 \times 512$ grid of cells. The top 100 rules with the highest complexity scores, which should correspond to rules with interesting behaviors, are then used for coarse-graining. We apply coarse-graining on grids of $4096 \times 4096$ cells, scaling the grid down by a factor of 8 , and compute the complexity metric also on the reduced grid. Figures reported in Table 1 are percentages of rules still considered interesting (above the selection threshold for the first step of the process) after coarse-graining. The higher this number is, the more a method is able to con- (a)

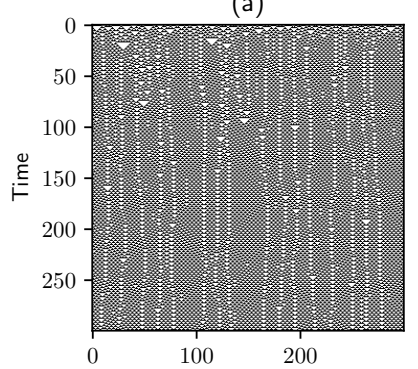

(c)



(b)

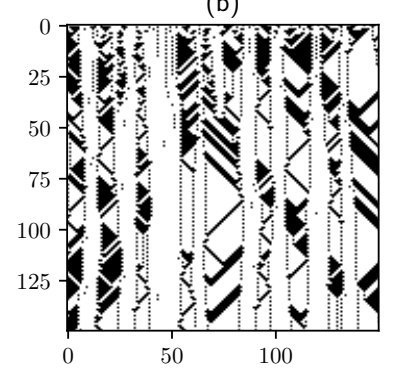

(d)

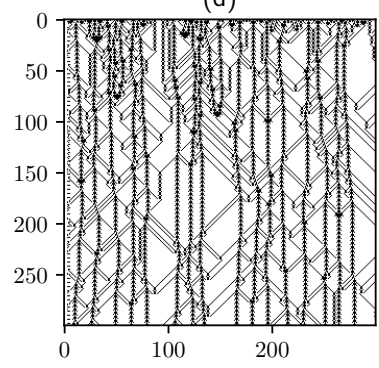

Figure 6: Space-time diagrams for rule 54. (a) Spacetime diagram of standard rule 54, starting from a random position. (b) Filtered domain with our frequency coarsegraining (even). (c) Particles filtered from the domains in (b) using our method. (d) Domain boundaries computed using computational mechanics (Hanson and Crutchfield, 1997). Please note that particles are detected equally well using computational mechanics (d) and our (simpler) frequencybased method (c). Some close-by particle trails are merged using our method.

\begin{tabular}{cccc}
\hline $\begin{array}{c}\text { Local-averaging } \\
\text { baseline }\end{array}$ & K-Means & Histogram & Autoencoder \\
\hline $19.3 \%$ & $40.4 \%$ & $82.4 \%$ & $84.2 \%$ \\
\hline
\end{tabular}

Table 1: Experimental results - Percentage of rules classified as interesting after reduction with our 3 proposed methods (K-means, Histogram, Autoencoder), compared to a local averaging baseline.

serve complex and interesting behaviors after the reduction.

Results in Table 1 suggest that using our proposed methods seems largely beneficial for studying complexity in large systems. Histogram and autoencoder methods are superior to downscaling using k-means and local averaging. This could be attributed to the fact that contrary to the latter two, the histogram and autoencoder both represent well anomalies (rare events). This is because rare events are explicitly captured and kept by the histogram method. They also represent useful information that may be kept for the reconstruction using the autoencoder. 

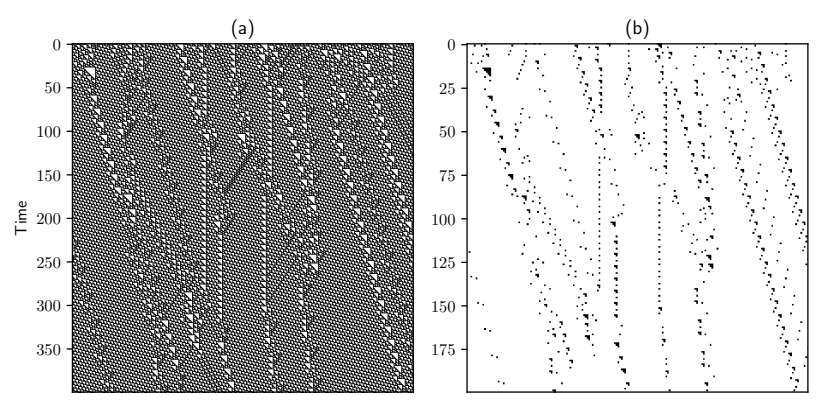

Figure 7: Spate-time diagram of rule 110 (a) and filtered particles using our histogram-based coarse-graining (b). Structures propagating in time (vertical axis) and space (horizontal axis) become clearly visible in (b) as vertical and diagonal lines.

\section{Discussion}

Downscaling by local averaging is not an effective solution to the coarse-graining problem for several reasons. In particular, it tends to favor the majority state in a supercell because of the averaging effect. Thin structures spanning only few cells placed on a uniform background are likely to disappear after coarse-graining although they may still be relevant with respect to the large-scale patterns. The histogrambased method explicitly encodes those more rare events in a supercell, even if their size is relatively small compared to the supercell size.

Figure 8 is a qualitative comparison of coarse-graining methods. This cellular automaton was selected from the experimental dataset. When simulated on large grids, it generates large linear structures that are 4-cells wide. These structure disappear after downscaling by averaging because the background dominates the average. Other methods correctly highlight these structures when downscaling the grids by a factor of 8 . In Figure 9, we show another rule that was selected for its high complexity score at multiple coarsegraining scales from our dataset. The CA has significantly different dynamics depending on the chosen scale. Example $9 \mathrm{a}$ is a spontaneously occurring stable oscillating glider with period 3. Large structures emerge from these simple gliders when observing large grids. The online project page $e^{2}$ shows animated example for Figure 9, emphasizing the advantage of using coarse-graining for visualization.

A crucial advantage of the frequency histogram method is its speed and ease of implementation compared to autoencoders. Other than a few hyper-parameters for partitioning the histogram, no training or tuning is needed to produce the coarse-grained output.

$\sqrt[2]{\text { https://hugocisneros.com/ }}$ ALIFE-Paper-2020/

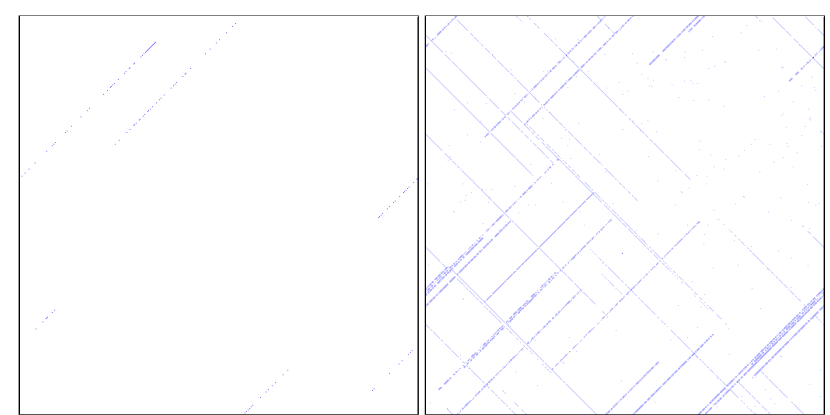

(a) Downscaling by averaging

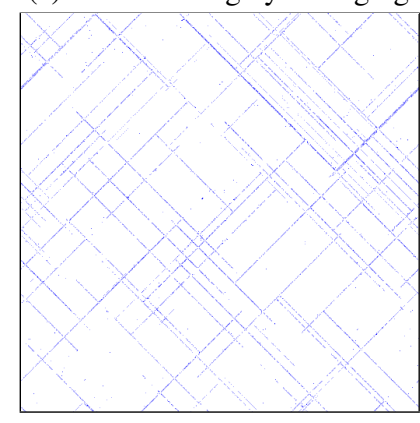

(c) K-means (b) Histogram

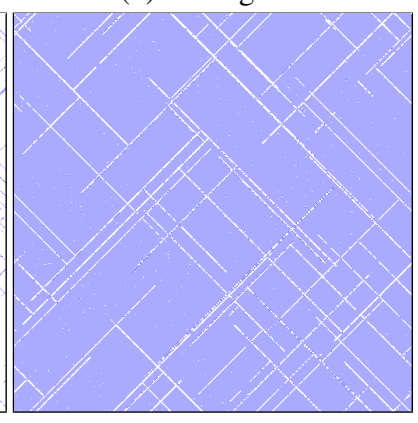

(d) Autoencoder
Figure 8: Qualitative comparison of coarse-graining methods. Simulations are on grids of $4096 \times 4096$ cells coarse-grained to $512 \times 512$. Lines are barely visible with downscaling $8 \mathrm{a}$, but are visible in $8 \mathrm{~b} \quad 8 \mathrm{~d}$. Coarse-graining helps visualize linear structures that would be hard to see otherwise.

\section{Conclusion}

We intend to use these coarse-graining methods to find cellular automata (CA) which exhibit interesting behaviors at multiple scales. Figure 9 shows an example of such a CA. We observe various dynamics depending on the scale, from simple oscillating gliders to large wave-like patterns composed of thousands of gliders. It demonstrates that observing multi-scale behaviors within those automata is possible. The existence of 2D cellular automata with disordered behaviors at the smallest level but organized at coarser scales, similar to hidden patterns in rule 18 , would also be of great interest.

Cellular automata are powerful computational models. Some of them have been shown to be Turing-complete, and can thus be expected to support arbitrarily complex computations (Berlekamp et al., 2001; Cook, 2004). Naturally, most interesting CAs spontaneously generate a fraction of available computations at a time, usually supporting a few stable oscillators or moving structures. Proofs of universality for these CAs required careful design of computational devices out of these stable oscillators and structures, resulting in very brittle and inefficient universal computers. In practise, only elementary functions - such as density clas- 


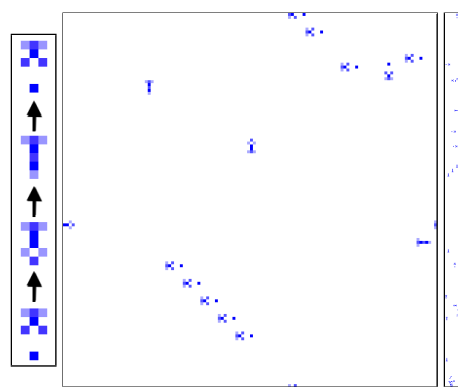

(a)

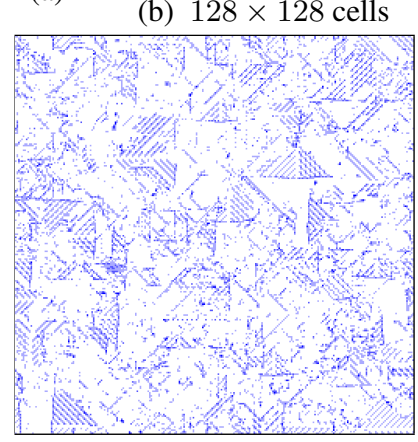

(d) $2048 \times 2048$ cells coarsegrained to $256 \times 256$.

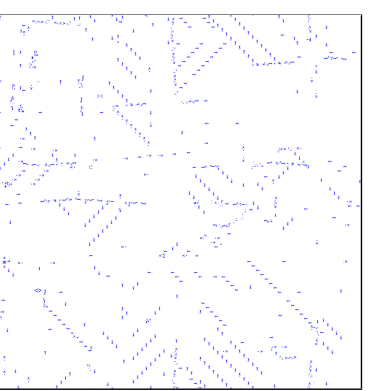

(c) $512 \times 512$ cells

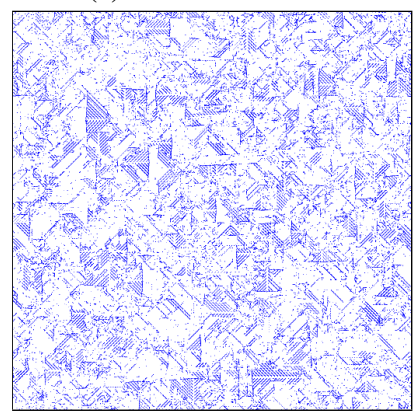

(e) $4096 \times 4096$ cells coarsegrained to $256 \times 256$.
Figure 9: Changing CA dynamics at multiple scales. (a) shows a single glider, oscillating between 3 positions. Such gliders emerge spontaneously from a random initialization of a small grid as shown in (b). When scaling the grid up, trails of gliders begin to appear, creating moving straight and diagonal lines as shown in (c). Scaling-up even more, individual gliders are not visible anymore, as shown in (d). In an even larger grid, shown in (e), many more triangular-shaped waves travel and collide with each other. Please note that (d) and (e) are coarse-grained to $256 \times 256$, otherwise the patterns are not visible.

sification, binary addition, etc. — can be implemented. This requires searching for CA rules specifically targeted at a particular function (Mitchell et al., 1996; Wolfram, 2002; Sapin et al. 2003). Hierarchies are central to naturally occurring complex phenomena (Simon, 1962), and may be required for robust and complex processes to emerge in CAs.

Viewing space-time diagrams of cellular automata is akin to visualizing a foreign computer design. Cellular automata are manipulating information, registers and instructions in parallel in the form of cell states. We believe visualization tools proposed in this paper can help understand computations in those unconventional computers. By reducing available information to its essential parts, we attempt to distill the content of the space-time diagram with as little prior information as possible. Future work could focus on identifying some known simple computational primitives within cellular automata and understanding how our visualization can help to find them.
These methods also enable apprehending large grid sizes for which even image processing algorithms begin to show limitations. Complexity metrics and CA classification techniques can be extended to these reduced large grids and could lead to the discovery of CAs with - similar to life and physical processes - significantly different dynamics at multiple scales that could in turn be a basis for artificial evolution.

Acknowledgements This work was partially supported by ERC grant LEAP No. 336845, CIFAR Learning in Machines \& Brains program and the EU Structural and Investment Funds, Operational Program Research, Development and Education under the project IMPACT (reg. no. CZ.02.1.01/0.0/0.0/15003/0000468).

\section{References}

Anderson, P. W. (1972). More Is Different. Science, 177(4047):393-396.

Baldi, P. and Hornik, K. (1989). Neural networks and principal component analysis: Learning from examples without local minima. Neural Networks, 2(1):53-58.

Berlekamp, E. R., Conway, J. H., and Guy, R. K. (2001). Winning Ways for Your Mathematical Plays. A.K. Peters, Natick, Mass, 2nd ed edition.

Boccara, N., Nasser, J., and Roger, M. (1991). Particlelike structures and their interactions in spatiotemporal patterns generated by one-dimensional deterministic cellular-automaton rules. Physical Review A, 44(2):866-875.

Cisneros, H., Sivic, J., and Mikolov, T. (2019). Evolving Structures in Complex Systems. In 2019 IEEE Symposium Series on Computational Intelligence (SSCI), pages 230-237, Xiamen, China. IEEE.

Cook, M. (2004). Universality in Elementary Cellular Automata. Complex Systems, page 40.

Crutchfield, J. P. and Hanson, J. E. (1993). Turbulent pattern bases for cellular automata. Physica D: Nonlinear Phenomena, 69(3-4):279-301.

Eloranta, K. and Nummelin, E. (1992). The kink of cellular automaton rule 18 performs a random walk. Journal of Statistical Physics, 69(5-6):1131-1136.

Floyd, R. W. and Steinberg, L. S. (1976). An adaptive algorithm for spatial gray scale. Journal of the Society for Information Display, 17:75-77.

Gardner, M. (1970). Mathematical Games. Scientific American, 223(4):120-123.

Gosper, R. (1984). Exploiting regularities in large cellular spaces. Physica D: Nonlinear Phenomena, 10(1-2):75-80.

Grassberger, P. (1986). Toward a quantitative theory of selfgenerated complexity. International Journal of Theoretical Physics, 25(9):907-938.

Hamming, R. W. (1950). Error Detecting and Error Correcting Codes. Bell System Technical Journal, 29(2):147-160. 
Hanson, J. E. and Crutchfield, J. P. (1992). The attractor-basin portrait of a cellular automaton. Journal of Statistical Physics, 66(5-6):1415-1462.

Hanson, J. E. and Crutchfield, J. P. (1997). Computational mechanics of cellular automata: An example. Physica D: Nonlinear Phenomena, 103(1):169-189.

Hinton, G. E. (1989). Connectionist learning procedures. Artificial Intelligence, 40(1-3):185-234.

Ingólfsson, H. I., Lopez, C. A., Uusitalo, J. J., de Jong, D. H., Gopal, S. M., Periole, X., and Marrink, S. J. (2014). The power of coarse graining in biomolecular simulations. Wiley Interdisciplinary Reviews: Computational Molecular Science, 4(3):225-248.

Israeli, N. and Goldenfeld, N. (2004). On computational irreducibility and the predictability of complex physical systems. Physical Review Letters, 92(7):074105.

Israeli, N. and Goldenfeld, N. (2006). Coarse-graining of cellular automata, emergence, and the predictability of complex systems. Physical Review E, 73(2):026203.

Kmiecik, S., Gront, D., Kolinski, M., Wieteska, L., Dawid, A. E., and Kolinski, A. (2016). Coarse-Grained Protein Models and Their Applications. Chemical Reviews, 116(14):7898-7936.

Kramer, M. A. (1991). Nonlinear principal component analysis using autoassociative neural networks. AIChE Journal, 37(2):233-243.

Langton, C. G. (1984). Self-reproduction in cellular automata. Physica D: Nonlinear Phenomena, 10(1):135-144.

Levitt, M. and Warshel, A. (1975). Computer simulation of protein folding. Nature, 253(5494):694-698.

Martinez, G. J., Adamatzky, A., and McIntosh, H. V. (2014). Complete characterization of structure of rule 54. Complex Systems, 23(3).

Mitchell, M., Road, H. P., Das, R., and Box, P. O. (1996). Evolving Cellular Automata with Genetic Algorithms: A Review of Recent Work. In Proceedings of the First International Conference on Evolutionary Computation and Its Applications, page 14.

Pivato, M. (2007). Spectral domain boundaries in cellular automata. arXiv:math/0507091.

Potoyan, D. A., Savelyev, A., and Papoian, G. A. (2013). Recent successes in coarse-grained modeling of DNA: Coarsegrained modeling of DNA. Wiley Interdisciplinary Reviews: Computational Molecular Science, 3(1):69-83.

Rupe, A. and Crutchfield, J. P. (2018). Local Causal States and Discrete Coherent Structures. Chaos: An Interdisciplinary Journal of Nonlinear Science, 28(7):075312.

Sapin, E., Bailleux, O., and Jean-Jacques, C. (2003). Research of a Cellular Automaton Simulating Logic Gates by Evolutionary Algorithms. In Goos, G., Hartmanis, J., van Leeuwen, J., Ryan, C., Soule, T., Keijzer, M., Tsang, E., Poli, R., and Costa, E., editors, Genetic Programming, volume 2610, pages 414-423. Springer Berlin Heidelberg, Berlin, Heidelberg.
Shalizi, C. R., Haslinger, R., Rouquier, J.-B., Klinkner, K. L., and Moore, C. (2006). Automatic filters for the detection of coherent structure in spatiotemporal systems. Physical Review $E, 73(3)$.

Shalizi, C. R., Shalizi, K. L., and Haslinger, R. (2004). Quantifying Self-Organization with Optimal Predictors. Physical Review Letters, 93(11):118701.

Simon, H. A. (1962). The Architecture of Complexity. Proceedings of the American Philosophical Society, 106(6):467-482.

Soler-Toscano, F., Zenil, H., Delahaye, J.-P., and Gauvrit, N. (2014). Calculating Kolmogorov Complexity from the Output Frequency Distributions of Small Turing Machines. PLOS ONE, 9(5):e96223.

Von Neumann, J. and Burks, A. W. (1966). Theory of selfreproducing automata. IEEE Transactions on Neural Networks, 5(1):3-14.

Wolfram, S. (2002). A New Kind of Science. Wolfram Media, Champaign, IL.

Wuensche, A. (1999). Classifying cellular automata automatically: Finding gliders, filtering, and relating space-time patterns, attractor basins, and the Z parameter. Complexity, 4(3):47-66.

Wuensche, A. (2011). Exploring Discrete Dynamics. Luniver Press.

Zenil, H. (2010). Compression-Based Investigation of the Dynamical Properties of Cellular Automata and Other Systems. Complex Systems, 19(1).

Zenil, H., Soler-Toscano, F., Delahaye, J.-P., and Gauvrit, N. (2015). Two-dimensional Kolmogorov complexity and an empirical validation of the Coding theorem method by compressibility. PeerJ Computer Science, 1:e23. 